\title{
Securing Content Sharing over ICN
}

\author{
Nikos Fotiou* \\ George C. Polyzos*,\# \\ * Mobile Multimedia Lab, Dept. of Informatics \\ School of Information Sciences and Technology \\ Athens University of Economics and Business \\ Evelpidon 47A, 11362 Athens, Greece \\ \#Dept. of Computer Science and Engineering \\ Jacobs School of Engineering \\ University of California, San Diego \\ La Jolla, CA 92093-0404, USA \\ fotiou@aueb.gr \\ polyzos@acm.org
}

\begin{abstract}
The emerging Information-Centric Networking (ICN) paradigm is expected to facilitate content sharing among users. ICN will make it easy for users to appoint storage nodes, in various network locations, perhaps owned or controlled by them, where shared content can be stored and disseminated from. These storage nodes should be (somewhat) trusted since not only they have (some level of) access to user shared content, but they should also properly enforce access control. Traditional forms of encryption introduce significant overhead when it comes to sharing content with large and dynamic groups of users. To this end, proxy re-encryption provides a convenient solution. In this paper, we use Identity-Based Proxy Re-Encryption (IBPRE) to provide confidentiality and access control for content items shared over ICN, realizing secure content distribution among dynamic sets of users. In contrast to similar IB-PRE based solutions, our design allows each user to generate the system parameters and the secret keys required by the underlay encryption scheme using their own Private Key Generator, therefore, our approach does not suffer from the key escrow problem. Moreover, our design further relaxes the trust requirements on the storage nodes by preventing them from sharing usable content with unauthorized users. Finally, our scheme does not require out-of-band secret key distribution.
\end{abstract}

Permission to make digital or hard copies of all or part of this work for personal or classroom use is granted without fee provided that copies are not made or distributed for profit or commercial advantage and that copies bear this notice and the full citation on the first page. Copyrights for components of this work owned by others than ACM must be honored. Abstracting with credit is permitted. To copy otherwise, or republish, to post on servers or to redistribute to lists, requires prior specific permission and/or a fee. Request permissions from permissions@acm.org.

ACM-ICN '16, September 26-28, 2016, Kyoto, Japan

(C) 2016 ACM. ISBN $978-1-4503-4467-8 / 16 / 09 \ldots \$ 15.00$

DOI: http://dx.doi.org/10.1145/2984356.2984376

\section{CCS Concepts}

-Networks $\rightarrow$ Network security; Security protocols; Middle boxes / network appliances; Network architectures; Network protocols; $\bullet$ Security and privacy $\rightarrow$ Security protocols; Authentication; Access control; Authorization; Cryptography; Security services; Network security;

\section{Keywords}

Identity-based encryption, Proxy re-encryption, ContentCentric Networking, Information-Centric Networking, Named-Data Networking

\section{INTRODUCTION}

Internet users regularly utilize web-based systems to share content with others. These systems provide facilities that allow content owners to store shared items online, as well as, to define access control policies. Authorized third parties can access the shared content, even if the content owner is disconnected. The emerging Information-Centric Networking (ICN) paradigm can be an excellent technology for implementing a content sharing system. Being information oriented, ICN, can achieve significant gains in terms of network performance and offers opportunities for innovative security solutions.

An important aspect of content sharing systems is that they should be highly trusted as they have access to user content and they are responsible for enforcing access control policies. Encrypting content before uploading it to a storage service can assuage most of the security and privacy concerns. Nevertheless, traditional forms of encryption are not convenient for sharing content with large and dynamic groups of users, as they require complex key management systems and pose significant communication and/or storage overhead when a user is added or removed from the list of authorized users. However, the same goal can be achieved more efficiently by using Proxy Re-Encryption (PRE) 11. PRE allows an entity, to transform a ciphertext computed 
under Alice's public key into one that can be decrypted by Bob's secret key, without having access neither to Alice's or Bob's secret keys, nor to the un-encrypted content. Therefore, by using PRE, a content owner can encrypt each content item only once and provide the storage node with the proper re-encryption keys, as well as, with the desired access control polices. In this setup the storage node needs to only be trusted to re-encrypt content only for authorized users (as determined by the access control policies) for proper system operation. But even if it fails to properly perform the re-encryption, no usable content is revealed.

In this paper, we use Identity-Based Proxy ReEncryption (IB-PRE) to implement a security and access control system for ICN-based content sharing and introduce the following two constructions.

Our first construction is a traditional IB-PRE-based system, with the added advantage that it allows users to use their own Private Key Generators (PKG), i.e., each user is able to generate by himself his secret key. This is a significant advantage compared to existing systems where a centralized PKG generates the keys for all users and therefore these systems suffer from the key escrow problem, i.e., PKGs know the private keys of the users and therefore they can impersonate them.

Our second construction improves on the first by relaxing the requirement for semi-trusted proxies, in the sense that our scheme is secure even if the storage node does not respect access control policies. With this construction a storage node can re-encrypt a content item only for authorized users.

In addition to these constructions, we provide a framework for defining and managing simple access control policies, as well as, an authentication protocol that can be used for user-to-storage node authentication as well as for content-to-user authentication, i.e., it allows end-users to verify that they received the desired content item from an authorized storage node. Our schemes do not require out-of-band secret key distribution and have very low overhead.

The remainder of this paper is organized as follows. In Section 2 we review identity-based encryption and proxy re-encryption and we discuss related work in the area. In Section 3 we detail our system design. In Section 4 we present our implementation and we evaluate our solution. In Section 5 we discuss various design choices and alternatives and the related tradeoffs. Finally, in Section 6 we conclude with a summary of the contributions of this paper.

\section{BACKGROUND \& RELATED WORK}

\subsection{Identity-Based Encryption}

An Identity-Based Encryption (IBE) scheme is a public key encryption scheme in which an arbitrary string can be used as a public key. An IBE scheme is specified by four algorithms: Setup, Extract, Encrypt and
Decrypt.

- Setup: it is executed by a Private Key Generator (PKG). It takes as input a security parameter $k$ and returns a master-secret key $(M S K)$ and some system parameters $(S P)$. The $M S K$ is kept secret by the PKG, whereas $S P$ are made publicly available.

- Extract: it is executed by a PKG. It takes as input $S P, M S K$, and an arbitrary string $I D$, and returns a secret key $S K_{I D}$.

- Encrypt: takes as input an arbitrary string $I D$, a message $M$, and $S P$, and returns a ciphertext $C_{I D}$

- Decrypt: takes as input $C_{I D}$, the corresponding private decryption key $S K_{I D}$, and returns $M$.

Therefore, using IBE and providing that $S P$ are known, it is possible to encrypt some plaintext using an arbitrary $I D$ as the public key. The entity that holds the $S K$ that corresponds to this $I D$ can decrypt the ciphertext.

\subsection{Proxy Re-Encryption}

A Proxy Re-Encryption (PRE) scheme is a scheme in which a third semi-trusted party, called, is allowed to alter a ciphertext, encrypted with the public key of a user $A$ (the delegator), in a way that another user $\mathrm{B}$ (the delegatee) can decrypt it with her own appropriate key (i.e., in most cases her secret private key). Green and Ateniese [11] implemented an Identity-Based Proxy Re-Encryption (IB-PRE) scheme that specifies two new algorithms, RKGen and Reencrypt, in addition to the IBE algorithms already discussed.

- RKGen: it is executed by the owner of a public key $I D 1$. It takes as input $S P$, his secret key $S K_{I D 1}$, and an identity $I D 2$ and generates a (public) reencryption key $R K_{I D 1 \rightarrow I D 2}$.

- Reencrypt: it is executed by a third semi-trusted party. It takes as input $S P$, a re-encryption key $R K_{I D 1 \rightarrow I D 2}$, and a ciphertext $C_{I D 1}$ and outputs a new ciphertext $C_{I D 2}$.

Figure1 gives an example of a complete IBE-PRE transaction. In this figure, initially the PKG generates the $M S K$ and the $S P$ and makes the $S P$ publicly available (step 1). Then it extracts $S K_{I D 1}$ and $S K_{I D 2}$ and distributes them to the corresponding users (step 2). Another user creates a ciphertext using as a public key the string $I D 1$ and stores it in a storage node (step $3)$. The user that owns $I D 1$ creates a re-encryption key $R K_{I D 1 \rightarrow I D 2}$ and sends it to the storage node. The node, re-encrypts $C_{I D 1}$ using $R K_{I D 1 \rightarrow I D 2}$ and generates $C_{I D 2}$. The user that owns $I D 2$ is now able to decrypt the new ciphertext. The storage node learns nothing about the contents of the ciphertext or the secret keys of the users. 


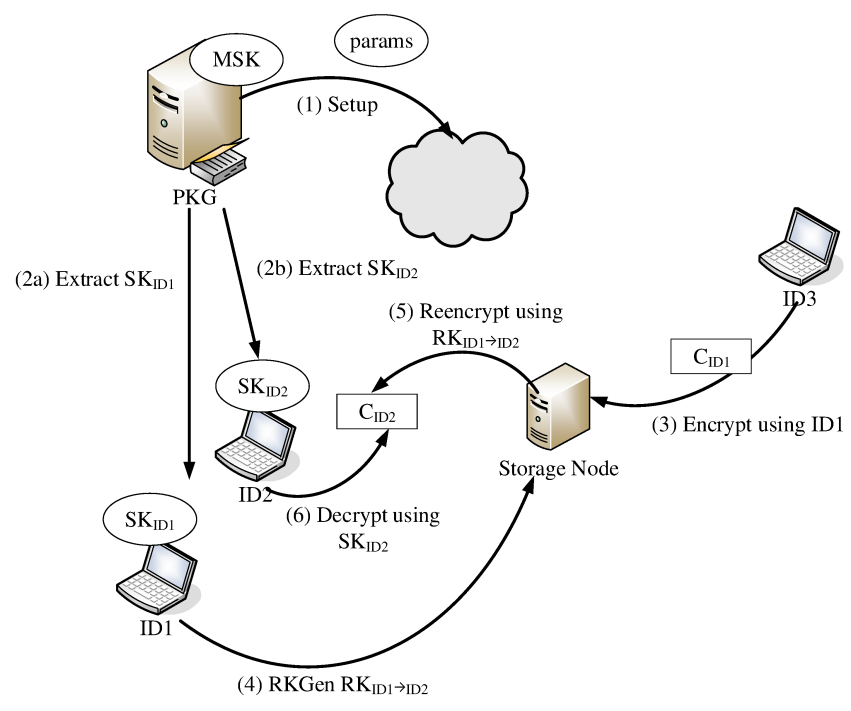

Figure 1: IBE-PRE example

\subsection{Related work}

Early attempts to secure distributed content sharing systems suffered from key management problems. In these systems (e.g., Plutus 17]) each content item is encrypted with a symmetric encryption key. Then this key has to be (securely) delivered individually, to each authorized user. Therefore, a large number of keys has to be stored and transferred to storage nodes. Our system exhibits much lower communication and storage overhead and uses simpler key management.

Advances in cryptography allowed the development of more efficient systems. A cryptographic scheme that has received significant attention by the research community is Attribute Based Encryption (ABE). ABE allows the specification of "attributes" that a user should have in order to decrypt a ciphertext. This is achieved by associating attributes with private keys. In addition, $\mathrm{ABE}$ allows the definition of simple access control policies using logical operators, e.g. a ciphertext can be decrypted by the users who have the 'CS' AND 'student' OR 'professor' attributes. ABE has been widely used for implementing contemporary systems with distributed content sharing capabilities (e.g., 3, 20]) and has received considerable attention by the ICN community (e.g., [14, [18, ,6]) . In these systems users use attributes to describe groups of trusted peers (e.g., 'friends', 'colleagues' etc.), then they encrypt their content items using a symmetric encryption key and encrypt this key using ABE.

The main drawback of these ABE systems is that removing a group member requires re-keying, i.e., the private keys that correspond to each attribute have to be regenerated and re-distributed. The same problem exists when a (compromised) private key has to be revoked. Jahid et al. 16] mitigate this problem by using the key revocation scheme presented in [19]. With this scheme the decryption of a ciphertext requires two keys: the private key that corresponds to the user's attributes and a secondary that is provided by a semi-trusted third party. The third party generates the secondary key for non revoked users using a secret key. Every time a user is removed from a group or a key has to be revoked, the only key that has to be updated is the secret key. In our work access control and user management are decoupled from the encryption of the shared content item, therefore, adding or removing a user from an access control policy does not involve any new encryption of the protected items. In addition, in ABE-based schemes content owners should securely distribute to other users the private keys that correspond to each attribute. In our design no out-of-band secret key distribution is required.

Zhang et al. 23 utilize the IBE scheme proposed by Boneh and Franklin 5] and the identity based signature scheme proposed by Hess 12 in order to provide namebased trust for the NDN architecture [15]. The identity used for the encryption of a content item in this scheme is either the name of the shared item, or the identity of the item's recipient. In the former case, the item's recipient learns the $S K$ that corresponds to the content name using out-of-band mechanisms. This solution is focused on content confidentiality and it is not efficient when it comes to access control. Our system combines proxy re-encryption with IBE and provides efficient access control in addition to content confidentiality.

Ateniese et al. 2] use IB-PRE for implementing a secure storage service. In this solution content items are stored in a semi-trusted storage node, encrypted with a symmetric key. The symmetric key is encrypted with the identity of the content owner. Every time a user requests access to a content item, the storage node requests from an access control server to create a re-encryption key, which is used for re-encrypting the symmetric key in a way that the content requester can decrypt it. The proposed solution uses a single $P K G$. This solution is used in an ICN context by Wood and Uzun 22 to implement a DRM-like solution for the CCN architecture as well as by Zheng et al. 24 to implement an access control mechanism for ICN. Our work extends these previous works by considering userspecific PKGs. Moreover, in these prior solutions, once the storage node learns the re-encryption key for a user, it may use it to re-encrypt all other shared items of the content owner, no matter whether individual users are authorized to access them or not. Our solution includes a construction that mitigates this problem.

\section{SYSTEM DESIGN}

\subsection{Inter-domain IB-PRE}

IB-PRE solutions usually assume that delegators and delegatees belong to the same administrative domain, therefore, they share the same PKG. This setup in many cases is not realistic. Moreover, given that PKGs know 
the users' secret keys, grouping many users under the same PKG raises security concerns. All these problems can be mitigated using Inter-domain IB-PRE 21. Inter-domain IB-PRE allows a $3^{\text {rd }}$ party to transform a ciphertext computed using an $I D$ and the $S P$ of a domain $A$ into a ciphertext intended for an $I D$ and the $S P$ belonging to another domain $B$. The Green-Ateniese scheme implements Inter-domain IB-PRE by allowing the usage of the $S P$ of the delegatee's domain as input to the $R K G e n$ algorithm ${ }^{1}$

\subsection{Setup}

Our system considers two types of users: content owners and content subscribers. All users are uniquely identified by an identity. The form and the semantics of this identity are user specific (e.g., an email address, a domain name, or a real world name). Moreover, diverse identity types may co-exist in the same instance of our system. Each user maintains his own PKG which is used to generate the user specific $S P$, as well as, the $S K$ that corresponds to his identity. A resolution service that maps identities to $S P \mathrm{~s}$ is assumed. Such a service could be implemented using DANE [13], Blockchains [10], Keyservers, third party directories, or even out-of-band mechanisms. Owners appoint (or own) storage nodes, where lists of known subscribers, access control policies, and shared content are stored. Storage nodes act as ICN publishers and are responsible for enforcing access control policies and for forwarding (encrypted) content items to authorized subscribers. Shared content items are identified by ICN routable identifiers, which are "advertised" in the network by the storage nodes using standard ICN procedures.

It should be noted that our system does not consider publisher (i.e. storage node) identities, since subscribers are interested in receiving a piece of content no matter its location. In section 3.3 .3 we present a protocol that allows a storage node to prove to a subscriber that it is authorized to store a particular content item.

\subsection{A first construction}

Each content owner maintains at a storage node three data structures: a table of Known Subscribers, a table of Access Control Policies, and a table of Shared Content. The table of Known Subscribers contains rows of the form [Identity, $S P, R K]$, where Identity is the identity of a subscriber known to the content owner, $S P$ is the $S P$ of that subscriber, and $R K$ is the re-

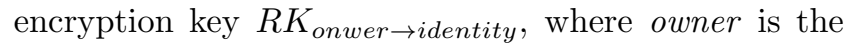
identity of the content owner and identity is the identity located in the first column of the row. The table of Access Control Policies contains rows of the form [Policy, List<Identity $>$ ], where Policy is an identifier

\footnotetext{
1 As a matter of fact, the Green-Ateniese scheme can even transform an IBE ciphertext into an RSA ciphertext (see section 5 of $[11]$ ).
}

Known Subscribers

\begin{tabular}{lll}
\hline Identity & SP & RK \\
\hline user@domain.com & $\mathrm{SP}_{\text {user@domain.com }}$ & $\mathrm{RK}_{\text {owner } \rightarrow \text { user@domain.com }}$ \\
Nikos Fotiou & $\mathrm{SP}_{\text {Nikos Fotiou }}$ & $\mathrm{RK}_{\text {owner } \rightarrow \text { Nikos Fotiou }}$ \\
mm.aueb.gr & $\mathrm{SP}_{\text {mm.aueb.gr }}$ & $\mathrm{RK}_{\text {owner } \rightarrow \text { mm.aueb.gr }}$ \\
\hline Access Control Policies & & \\
\hline $\begin{array}{l}\text { Policy } \\
\text { Friends }\end{array}$ & Identities \\
\hline Colleagues & user@domain.com, Nikos Fotiou \\
\hline Shared Content & mm.aueb.gr \\
\hline Item & Policy & Key \\
\hline Holidays.jpg & Friends & $\mathrm{C}_{\text {owner }}($ Key1) \\
\hline Balance.xls & Colleagues & $\mathrm{C}_{\text {owner }}($ Key2) \\
\hline
\end{tabular}

Figure 2: Storage node components with the first construction

of the access control policy and List $<$ Identity $>$ is a list of subscriber identities that abide by that access control policy. These identities should also exist in the Known Subscribers table. Finally, the table of Shared Content contains rows of the form [Item, Policy, $\left.C_{\text {owner }}(K)\right]$, where Item is the identifier of the (shared) content item, Policy is the policy of the access control policy that protects this item, and $C_{\text {owner }}(K)$ is the encryption of the symmetric encryption key $K$, generated as described in the following section.

For each content item she wants to share, a content owner, selects a symmetric encryption key $K$, encrypts the item using a symmetric encryption algorithm Enc, and encrypts $K$ using the Encrypt IBE algorithm with input her $S P$ and her identity, producing this way $C_{\text {owner }}(K) 2^{2}$ Then she stores the content item in a storage node and populates its tables accordingly.

Figure 2 illustrates an instance of a storage node. As it can be seen, the Known Subscribers table of the node contains three subscriber identities. For each identity the corresponding re-encryption key has been created. Moreover, two access control policies have been created using the subscriber identities. Finally, two items have been added in the table of Shared Content.

\subsubsection{Content sharing with trusted subscribers}

A content item can be shared with some of the subscribers included in the table of Known Subscribers.

\footnotetext{
${ }^{2}$ In the Green-Ateniese scheme the message $M$ that is used in the Encrypt algorithm is an element of a group $G$ of prime order $q$. Therefore, to be precise, the content owner selects a random element $K$ in $G$ and uses a secure hash function $H: G \rightarrow\{0,1\}^{n}$ (where $n$ is sufficiently large, e.g., 128) to calculate $H(K)$, which is then used as a key by the symmetric encryption algorithm.
} 
A content owner should perform the following steps: (i) create an access control policy, assign to this policy a list of subscriber identities that can access the item, and update accordingly the storage node's Access Control Policies table, (ii) encrypt the item using a symmetric encryption key and encrypt this key using her identity, i.e., generate $C_{\text {owner }}(K)$, and (iii) copy the encrypted item in the storage node and update its Shared Content table.

\subsubsection{Content sharing with subscribers another known subscriber knows}

In many cases it is desirable to share a content item with subscribers another known subscriber knows, e.g., in terms of social networks with the 'friends' of my friend. In order to implement this functionality, wellknown policy identifiers can be used. Suppose, an owner with identity $A$ trusts a subscriber with identity $B$, and $A$ wants to share a content item with the 'colleagues' of $B$. In this case 'colleagues' is a well-known policy identifier. $A$ encrypts the item using symmetric encryption and encrypts the symmetric encryption key using IBE encrypt with input $B$ and $B^{\prime} s S P P^{3}$ I.e., $A$ creates $C_{B}(K)$ using $B^{\prime} s S P$. Then, the encrypted item, as well as $C_{B}(K)$, are stored in $B^{\prime} s$ storage node, and $B^{\prime} s$ storage node table of Shared Content is modified accordingly. It should be noted that since the symmetric encryption key is encrypted using $B$ as the public key and $B^{\prime} s S P, B$ can also access the content item.

\subsubsection{Endpoints authentication and secure chan- nel setup}

Our construction assures that subscribers that "lie" about their identity cannot decrypt the received content. Therefore, having a storage node blindly accepting a subscriber's claims about his identity can be a valid design choice. On the other hand it may be desirable to have communicating endpoints authenticated to each other. E.g., for preserving bandwidth by not sending content items to unauthorized subscribers, for hiding the existence of a content item from unauthorized subscribers, for hiding subscribers' interests from malicious storage nodes, etc., as well as to transmit control messages over a secure communication channel.

In the following we present a handshake protocol that takes place before any content request and has the following properties (i) it provides subscriber authentication, (ii) it provides a proof that a storage node is authorized to store a particular content item, and (iii) it enables the creation of an ephemeral symmetric encryption key that can be used for securing the communication channel between the two endpoints.

Let $U$ be the identity of a subscriber that wants to access an item $F$. Moreover, let $H$ be an keyed-Hash Message Authentication Code (HMAC) function, and Enc a symmetric encryption algorithm.

\footnotetext{
${ }^{3}$ Remember that $S P$ are publicly available.
}

step 0: The content owner uses the Extract IBE algorithm, generates $S K_{F}$, i.e, the secret key that corresponds to the item identifier, and stores this key to the storage node that hosts the item.

step 1: $U$ learns out-of-band the owner's $S P$ and generates a random number $r$, a key $h$ that is used by $H$, a key $k$ that is used by Enc, and a Diffie-Hellman parameter $D H_{U}$, and sends to the storage node the following subscription message:

$$
m s g 1: F, C_{F}(k), E n c_{k}\left(r, h, D H_{U}, U\right), H_{h}(M S G)
$$

where $C_{F}$ is a ciphertext generated using the IBE encrypt algorithm with input $F$ and $k$ and $H_{h}(M S G)$ is the output of $H$ using $h$ applied over the whole message. The content item identifier $F$ is included in the plaintext for two reasons: first, because it is required by the underlay ICN routing plane and, second, to help shared storage nodes to decide which $S K$ to use. (Note: this is similar to the Server Name Indication TLS extension [7].)

step 2: The storage node (Node) decrypts $C_{F}(k)$ (using $S K_{F}$ from step 0), then decrypts $E_{n c}\left(r, h, D H_{U}, U\right)$, and then verifies $H_{h}(M S G)$. If the verification succeeds, the storage node retrieves $U$ 's $S P$ from the Known Subscribers table, generates a random number $r^{\prime}$, a key $k^{\prime}$, and a Diffie-Hellman parameter $D H_{N o d e}$, and sends to $U$ :

$$
m s g 2: C_{U}\left(k^{\prime}\right), E_{n k^{\prime}}\left(r, r^{\prime}, D H_{\text {Node }}\right), H_{h}(M S G)
$$

step 3: $U$ verifies $H_{h}(M S G)$; if the verification succeeds, $U$ decrypts $C_{U}\left(k^{\prime}\right)$, then decrypts $E n c_{k^{\prime}}\left(r, r^{\prime}, D H_{\text {Node }}\right)$, and then verifies that $r$ is the same with that included in his first message. If the verification succeeds, $U$ calculates a secret key $s$ using $D H_{U}$ and $D H_{N o d e}$, and sends to the storage node:

$$
m s g 3: \operatorname{Enc}_{s}\left(r^{\prime}\right), H_{h}(M S G)
$$

step 4: The storage node verifies $H_{h}(M S G)$; if the verification succeeds, it calculates $s$, it decrypts $E n c_{s}\left(r^{\prime}\right)$, and examines if $r^{\prime}$ is the same with that included in its previous message.

At the end of this protocol, the subscriber has been authenticated to the storage node, the storage node has proven to the subscriber that it is authorized to host the desired content item, and both entities have established common encryption and HMAC keys.

Proof: $C_{F}$ can only be decrypted by an entity that knows $S K_{F}$. If $m s g 1$ and $m s g 2$ contain the same values for $r$ then the storage node knows $S K_{F}$. Similarly $C_{U}$ can only be decrypted by the owner of $S K_{U}$. If $m s g 2$ and $m s g 3$ contain the same value for $r^{\prime}$ then the subscriber knows $S K_{U}$. The hashes included in all messages protect the messages integrity. Moreover, providing that $U$ learns the correct storage node's $S P$, man in the middle attacks are not possible. 
As a next step, $U$ issues a new subscription message that includes in its payload a content request. The request is encrypted with $E N C_{s}$ and the hash of the ciphertext is calculated using $H_{h}$. Upon receiving the request, the storage node (i) retrieves the record that corresponds to the content identifier included in the request from the Shared Content table, (ii) retrieves the list of identities that abide by the access control policy used to protect the item in question from the Access Control Policies table, (iii) checks if $U$ is included in the list of identities retrieved during step (ii); if yes, (iv) it retrieves the re-encryption key that corresponds to $U$ from the Known Subscribers table, re-encrypts $C_{\text {owner }}(K)$ (i.e., the encrypted key found in the Shared Content table), and sends the new ciphertext along with the encrypted content item to $U$.

\subsection{A second construction: allowing un- trusted storage nodes}

In the first construction, and generally in most proxy re-encryption schemes, the storage node is trusted to perform the re-encryption process only for authorized subscribers. A malicious storage node, however, can use a re-encryption key to re-encrypt all $C_{\text {owner }}(K)$ ciphertexts, and make them accessible to a subscriber no matter whether the subscriber is authorized to access $K$. This attack can be mitigated using the following construction.

For each access control policy identifier, the content owner uses the Extract IBE algorithm to generate $S K_{\text {Policy }}$. Then, for each content item she wants to share, she selects a symmetric encryption key $K$, encrypts the item using a symmetric encryption algorithm and $K$, and encrypts $K$ using the Encrypt IBE algorithm with input her $S P$ and the identifier of the access control policy that protects the content item, producing this way $C_{\text {Policy }}(K)$.

The data structures of the storage nodes are now modified as follows. The table of Known Subscribers contains rows of the form [Identity, $S P]$. The table of Access Control Policies contains rows of the form $[$ Policy, List $<($ Identity, $R K)>]$, where List $\langle($ Identity,$R K)\rangle$ is a list of pairs of subscriber identities that abide by that access control policy and the corresponding re-encryption key $R K_{\text {Policy } \rightarrow \text { identiy }}$ generated using the $S K_{\text {Policy. Finally, the ta- }}$ ble of Shared Content contains rows of the form $\left[\right.$ Item, Policy, $\left.C_{\text {Policy }}(K)\right]$. Figure 3 illustrates an instance of a storage node based on the second construction.

When a subscriber $U$ subscribes to a content item, the storage node selects $R K_{P o l i c y} \rightarrow U$ from the Access Control Policies table to re-encrypt $C_{P o l i c y}(K)$. This re-encryption key can only be used to re-encrypt ciphertexts encrypted using Policy as the public key, i.e., ciphertexts that $U$ is authorized to access.

It should be noted that these constructions are compatible with each other, i.e., an owner that uses the first

\begin{tabular}{|c|c|c|c|}
\hline \multicolumn{3}{|c|}{ Known Subscribers } & \\
\hline \multicolumn{4}{|c|}{ Identity SP } \\
\hline \multicolumn{4}{|c|}{ user@domain.com SP $\mathrm{S}_{\text {user@domain.com }}$} \\
\hline \multicolumn{2}{|l|}{ Nikos Fotiou } & $\mathrm{SP}_{\text {Nikos Fotiou }}$ & \\
\hline \multicolumn{2}{|l|}{ mm.aueb.gr } & $\mathrm{SP}_{\text {mm.aueb.gr }}$ & \\
\hline \multicolumn{4}{|c|}{ Access Control Policies } \\
\hline Policy & \multicolumn{3}{|c|}{ Identities } \\
\hline Friends & & \multicolumn{2}{|c|}{$\begin{array}{l}\text { user@domain.com, } \mathrm{RK}_{\text {Friends } \rightarrow \text { user@domain.com }} \\
\text { Nikos Fotiou, RK } \mathrm{RK}_{\text {Friends } \rightarrow \text { Nikos Fotiou }}\end{array}$} \\
\hline Colleagues & \multicolumn{3}{|c|}{ mm.aueb.gr, $\mathrm{RK}_{\text {Colleagues } \rightarrow \mathrm{mm} . \text { aueb.gr }}$} \\
\hline \multicolumn{4}{|c|}{ Shared Content } \\
\hline \multicolumn{3}{|c|}{ Policy } & Key \\
\hline Holidays.jpg & \multicolumn{2}{|c|}{ Friends } & $\mathrm{C}_{\text {Friends }}($ Key1) \\
\hline Balance.xls & \multicolumn{2}{|c|}{ Colleagues } & $\mathrm{C}_{\text {Colleagues }}($ Key2) \\
\hline
\end{tabular}

Figure 3: Storage node components with the second construction

construction can share a content item with a subscriber that uses the second construction and vice versa.

\section{EVALUATION}

\subsection{Security evaluation}

The Green-Ateniese scheme has been proven to have the following security properties [11]: (i) it is chosenplaintext attack (CPA) secure, (ii) re-encryption key generation does not require any delegatee involvement, (iii) the entity that performs a re-encryption can not create new re-encryption keys from the existing ones, and (iv) re-encryption keys are unidirectional.

Each content item is encrypted using a different symmetric encryption key, therefore, the compromise of a symmetric encryption key would require a new encryption of the content item with another fresh key and the update of the corresponding entry in the Shared Content table. This is an inevitable overhead of all similar systems and it is due to the fact that public key encryption cannot be applied directly to the content item, because of its computational complexity. Nevertheless, if small items are considered, e.g., sensor measurements, news headlines, tweets, etc., it could be possible to relax the need for symmetric encryption.

Access control policy management in our system is orthogonal to content encryption. Let $A, B, C$ be three users, and suppose that $A$ has created an access control policy named 'friends' that includes $B$ and $C$. If $A$ decides to remove $B$ from this policy, he has simply to update the corresponding entry in the Access Control Policies table. No further action is required (e.g., no re-keying or re-encryption of a content item), provided that the storage node will not use any cached re- 
encryption key generated while $B$ was authorized. This is not the case with systems that incorporate access control policies in ciphertexts (e.g, ABE-based systems); in these systems the modification of an access control policy requires the re-encryption of the content item (or of the symmetric key that protects this item).

If the master secret key of user $A$ 's PKG is compromised, $A$ has to perform the following actions: (i) generate new $S P$, master secret key, and $S K \mathrm{~s}$, (ii) create fresh re-encryption keys, (iii) create fresh $C_{A}(K)$ (or $\left.C_{P o l i c y}(K)\right)$ ciphertexts, and (iv) update the corresponding storage node tables. Content items are not required to be encrypted again. Moreover, any other user that shares content with $A$ should update the corresponding re-encryption keys.

If the $S K$ of a user is compromised, the user has two options: either perform the same steps as in the case of master secret key loss, or change identity (e.g., using the serial number approach of $[9]$ ). With the latter option the user has only to update the re-encryption keys. Finally, when it comes to our second construction, in case of a $S K_{\text {Policy }}$ compromise, a user has again the same two options: either follow the procedure used for the master secret key loss, or change the name of the access control policy (the latter however may be harder for well known policy names). Again, if a policy is renamed, the corresponding re-encryption keys should be updated.

A storage node learns nothing about subscriber secret keys or the shared content. We now discuss the case of a storage node compromise. We distinguish two types of attackers: (a) third party attackers, and (b) attackers that abide by a certain access control policy (i.e., authorized by the access control policy). In the former case (i.e., attackers with no authorization at all), the attacker gains no information about the stored content, neither about the subscriber secret keys. In the latter case (i.e., attackers with authorization), if our first construction is used, the attacker is able to decrypt all content items by using the $R K_{\text {onwer } \rightarrow \text { identity }}$ re-encryption key included in the Known Subscribers table; if our second construction is used, the attacker is able to decrypt only the items protected by the so authorizing access control policy. In both cases, the attacker learns no information about the the secret keys of other users.

\subsection{Performance evaluation}

The IB-PRE algorithm of Green and Ateniese used in our system has been implemented ${ }^{4}$ using the Charm Crypto library [1. In order to achieve a security level equivalent to RSA with key size 1024 bits, the size of $S P$ is 2048 bits, the size of $C_{I D}(k e y)$ is 2048 bits, and the size of a re-encryption key is 3072 bits. In an Ubuntu 12.04 desktop machine running in a single core of an Intel i5-4440 3.1 GHz processor and with $2 \mathrm{~GB}$ of RAM, the creation of $C_{I D}(k e y)$, where key is a 128 bits sym-

\footnotetext{
${ }^{4}$ We used the first construction presented in $[11$.
}

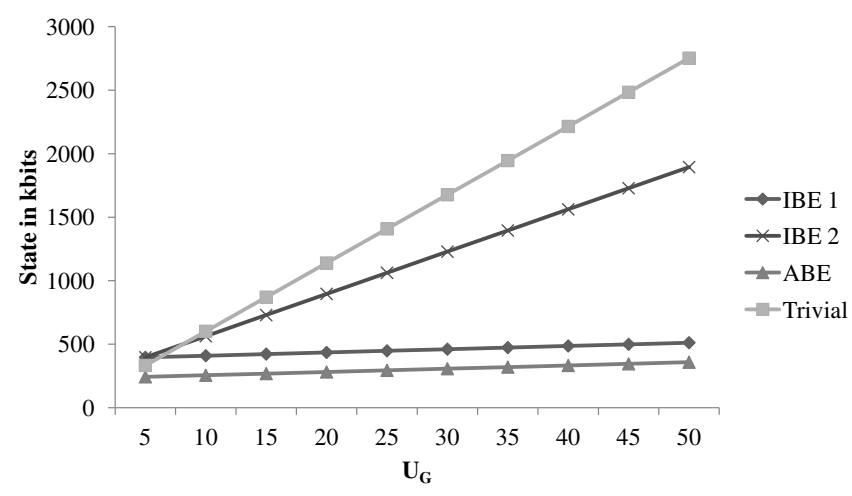

Figure 4: Storage overhead as a function of $U_{G}$, the number of subscribers per access control policy

metric encryption key, required $40 \mathrm{~ms}$; the creation of a re-encryption key required $20 \mathrm{~ms}$, the re-encryption of a ciphertext required $31 \mathrm{~ms}$, and the decryption of an IBE ciphertext required $28 \mathrm{~ms}$. These numbers are means of 20 runs of the same experiment; variation among experiments was negligible. It should be noted that IBE is used for encrypting, decrypting, and re-encrypting a symmetric encryption key, therefore these measurements are independent of the size of the content item.

We now compare the storage and communication overhead of our solution against a public key based trivial solution and an $A B E$ based solution. We do not consider the overhead of the handshake protocol described in section 3.3.3. In the case of the trivial solution, all users are equipped with a self-generated public/private key pair. Public keys are distributed using out-of-band mechanisms. Each content item is encrypted with a symmetric encryption key and this key is encrypted with the public keys of all subscribers that are allowed to access the item. In the ABE-based solution, we consider each policy as an attribute. Every content onwer generates public/private keys that correspond to attributes and distributes them accordingly. Each content item is encrypted with a symmetric key and this key is encrypted using a key that corresponds to a specific attribute; only subscribers that own the corresponding attribute keys can decrypt the symmetric key. Hence, we are considering $\mathrm{CP}-\mathrm{ABE}$ without "policy trees."

For our evaluation we consider the following scenario. A content owner knows $U$ subscribers and has grouped them in $G$ access control policies. Each policy contains $U_{G}$ subscribers. (A subscriber may belong to multiple policies.) Moreover, the owner shares $F$ content items, each of which is protected by a single access control policy.

\subsubsection{Storage overhead}

With our scheme, in addition to the encrypted content, a storage node maintains the following structures: 


\begin{tabular}{|c|c|c|}
\hline Symbol & Meaning & $\begin{array}{l}\text { Value } \\
\text { (bits) }\end{array}$ \\
\hline $\mid \overline{|I D|}$ & $\begin{array}{l}\text { Size of a subscriber identity } \\
\text { or of a content item identi- } \\
\text { fier }\end{array}$ & 256 \\
\hline$|S P|$ & Size of system parameters & 2048 \\
\hline$|S K a \rightarrow b|$ & Size of a re-encryption key & 3072 \\
\hline$\left|C_{\text {identity }}(K)\right|$ & $\begin{array}{l}\text { Size of a symmetric encryp- } \\
\text { tion key encrypted using } \\
\text { IBE }\end{array}$ & 2048 \\
\hline$|P K|$ & Size of public key & 1024 \\
\hline$|\operatorname{Enc}(K)|$ & $\begin{array}{l}\text { Size of an encrypted sym- } \\
\text { metric encryption key }\end{array}$ & 1024 \\
\hline$\left|A B E_{a t t r}(K)\right|$ & $\begin{array}{l}\text { Size of a symmetric encryp- } \\
\text { tion key encrypted using } \\
\text { ABE (we consider the con- } \\
\text { struction of }[4] \text { ) }\end{array}$ & 4096 \\
\hline
\end{tabular}

Table 1: Notation and parameters for storage overhead evaluation

a table of Known Subscribers, a table of Access Control Policies, and a table of Shared Content. In our evaluation scenario, and for our first construction, the storage node should maintain for $U$ identities $S P$ and re-encryption keys (i.e., the Known Subscribers table), $G \times U_{G}$ identities in the Access Control Policies table, and $F$ content item identifiers and encrypted symmetric encryption keys. For simplicity, we assume that content item identifiers and subscriber identities have the same size in bits. The storage overhead of our fist construction can then be calculated as follows:

$$
\begin{aligned}
S= & U \times(|I D|+|S P|+|S K a \rightarrow b|) \\
& +G \times U_{g} \times|I D| \\
& +F \times\left(|I D|+\left|C_{\text {owner }}(K)\right|\right)
\end{aligned}
$$

where $|I D|$ is the size of a subscriber identity (or of a content item identifier), $|S K a \rightarrow b|$ the size of a reencryption key, and $\left|C_{\text {owner }}(k e y)\right|$ the size of the encrypted symmetric encryption key. Table 1 contains this notation as well as the size of each field.

In our second construction re-encryption keys are stored in the Access Control Policies table instead of the Known Subscribers table. Therefore, the storage overhead can be calculated as follows:

$$
\begin{aligned}
S= & U \times(|I D|+|S P|) \\
& +G \times U_{g} \times(|I D|+|S K a \rightarrow b|) \\
& +F \times\left(|I D|+\left|C_{A C P}(K)\right|\right)
\end{aligned}
$$

When the trivial solution is used, then a storage node should maintain $U$ identities and public keys, $G \times U_{G}$ identities for the access control policies, $F$ content item identifiers, and $F \times U_{G}$ encryptions of the symmetric encryption key. Therefore, in this case the size of the

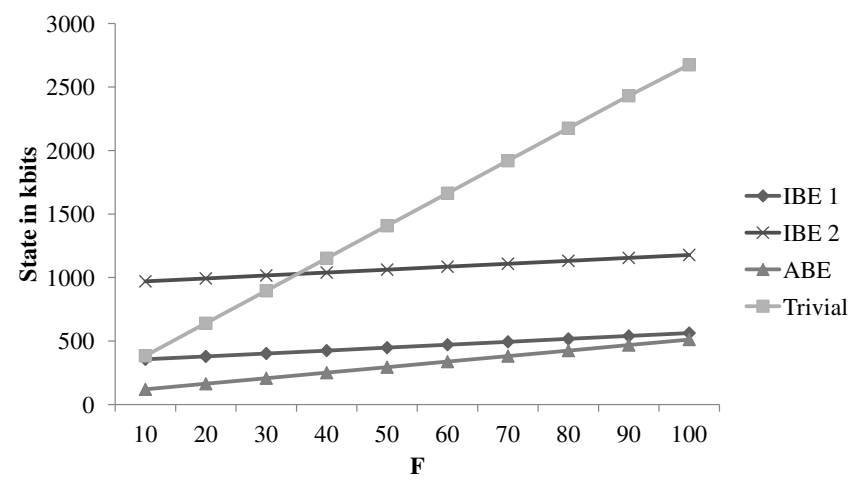

Figure 5: Storage overhead as a function of $F$, the number of content items shared by each owner

state maintained can be calculated as follows:

$$
\begin{aligned}
S= & U \times(|I D|+|P K|) \\
& +G \times U_{G} \times|I D| \\
& +F \times\left(|I D|+U_{G} \times|\operatorname{Enc}(K)|\right)
\end{aligned}
$$

where $|P K|$ is the size of a public key and $|\operatorname{Enc}(K)|$ is the size of the encrypted symmetric encryption key.

When the ABE-based solution is used, each policy is treated as an attribute, therefore the storage node should maintain $U$ identities, $G \times U_{G}$ attribute identifiers, and $F \times U_{G} \mathrm{ABE}$ encryptions of the symmetric encryption key. Therefore:

$$
\begin{aligned}
S= & U \times|I D| \\
& +G \times U_{G} \times|I D| \\
& +F \times\left(|I D|+\left|A B E_{a t t r}(K)\right|\right)
\end{aligned}
$$

where $\left|A B E_{a t t r}(K)\right|$ is the size of the symmetric encryption key encrypted using $\mathrm{ABE}$ and a single attribute. Figure 4 shows the storage overhead as a function of $U_{G}$. In this experiment $F$ is set to 50 . Figure 5 shows the storage overhead as a function of $F$. In this experiment $U_{G}$ is set to 25 . In both experiments $U$ is set to 50 .

\subsubsection{Communication overhead}

We now examine the communication overhead introduced when the right of a subscriber to access content is revoked. When our system is used, the only thing that has to be done is to remove the identity of that subscriber from the Access Control Policies table. Therefore, a content owner has to send a single message to the storage node containing the changes to the access control policies. With the trivial solution, a content owner should remove the identity of the revoked subscriber from the Access Control Policies table and also remove all encryptions of the symmetric encryption key generated using the public key of the revoked subscriber. Finally, with the ABE-based solution, when a subscriber is revoked, a content owner should remove the revoked subscriber from the Access Control Policies table, re- 
generate the encryptions of the symmetric encryption key (which otherwise the revoked subscriber could access), and send the new ciphertexts to the storage node.

\section{DISCUSSION}

Our design allows each user to maintain his own PKG. This feature protects the system from the key escrow problem and supports various other features based on the properties of IBE. For example, providing that a subscriber knows the $S P$ of a content owner, he can easily verify that a storage node is allowed to store a content item (identifier), since all cryptographic operations based on the item identifiers of the same owner use the same $S P$. Another advantage of supporting per-user PKG is that key revocation becomes easier: when per-user PKGs are used, users have the option to simply update their $S P$. However, each user maintaining his own PKG creates an additional overhead for content owners since they have to retrieve the $S P$ of their subscribers. In section 3.2 we mentioned solutions that can be used for secure $S P$ retrieval. In any case, our scheme does not prohibit the use of the same PKG among (a set of) users. In that case PKGs know the users' secret keys, but content owners only have to retrieve the $S P$ once for each set of users. Moreover, providing that there is a secure method for a storage node to verify the identity of a subscriber, the $S P$ of a subscriber (which are public) can be simply included in a message the subscriber sends to the node before any re-encryption.

The authentication protocol presented in section 3.3.3 allows subscribers to verify that a storage node is authorized to store a particular piece of content. This is achieved by encrypting a random number using the IBE encrypt algorithm with input the content item identifier. If a subscriber wants to receive multiple items from a storage node and for each content item she wishes to verify that the node is authorized to store it, then this protocol has to be executed before every request. This may result in increased network overhead and latency. An alternative approach could be to use the same prefix for a group of content item identifiers, and verify that a storage node is allowed to store this prefix. In that case, the subscriber should encrypt the random number using the prefix as input to the IBE encrypt algorithm. Then, the authentication protocol has to be repeated only once, when requesting items belonging to the same group. Of course, this construction requires content owners to generate the secret keys that correspond to identifier prefixes and provide them in the storage nodes.

A feature not yet implemented in our system is content item listing. Such listing function should respect the access control policies defined. Note that in the scheme as described here, access control policies are stored in the storage node and this may raise some privacy concerns; extensions to our design that use other trusted parties for storing and evaluating access control policies (similar to 8]) can be introduced to address these concerns. In that case the storage node would redirect subscribers requesting access to a content item to that trusted (third) party, which in turn would authenticate subscribers and provide the storage node with the appropriate re-encryption key.

Finally, our design has been independent of any particular ICN architecture and applicable to all. Only further optimizations, such as the prefix grouping discussed just above, depend on specific characteristics of the ICN architecture. The key aspects of ICN that are exploited by our design are the naming of individual information items by the content owner and their atomic treatment, at least at the level of users and storage nodes.

\section{CONCLUSION}

In this paper we presented a secure ICN-based content sharing system that leverages identity-based proxy re-encryption. Our scheme does not suffer from the key escrow problem, it does not require any pre-shared secret information, and it has low storage and network overhead. Moreover, we (1) presented an access control framework, (2) designed an IBE-based authentication protocol limiting storage node and network traffic overhead by unauthorized users, and (3) a construction that allows the use of untrusted storage nodes.

The security evaluation demonstrated that the scheme possesses the required properties and in particular that storage nodes do not learn anything about subscriber secret keys or the shared content. Even in the case of a storage node compromise, the outcome is reasonable and in particular no information about the secret keys of the users is obtained. The performance evaluation of the system illustrated the low storage and network overhead of the system and compared it to basic alternatives.

\section{ACKNOWLEDGMENTS}

This research was supported by the EU funded H2020 ICT project POINT, under contract 643990.

\section{REFERENCES}

[1] Akinyele, J. A., Garman, C., Miers, I., Pagano, M. W., Rushanan, M., Green, M., And Rubin, A. D. Charm: a framework for rapidly prototyping cryptosystems. Journal of Cryptographic Engineering 3, 2 (2013), 111-128.

[2] Ateniese, G., Fu, K., Green, M., And HoHENBERGER, S. Improved proxy re-encryption schemes with applications to secure distributed storage. ACM Transactions on Information and System Security 9, 1 (February 2006), 1-30.

[3] Baden, R., Bender, A., Spring, N., Bhattacharjee, B., And Starin, D. Persona: An online social network with user-defined 
privacy. ACM SIGCOMM Computer

Communication Review 39, 4 (August 2009), 135-146.

[4] Bethencourt, J., Sahai, A., and Waters, B. Ciphertext-policy attribute-based encryption. In IEEE Symposium on Security and Privacy (May 2007), pp. 321-334.

[5] Boneh, D., and Franklin, M. Identity-based encryption from the weil pairing. In Advances in Cryptology - CRYPTO 2001, Proc. Annual International Cryptology Conference, J. Kilian, Ed., vol. 2139 of Lecture Notes in Computer Science. Springer, 2001, pp. 213-229.

[6] DA Silva, R. S., ANd Zorzo, S. D. An access control mechanism to ensure privacy in named data networking using attribute-based encryption with immediate revocation of privileges. In Proc. Annual IEEE Consumer Communications and Networking Conference (January 2015), pp. 128-133.

[7] Eastlake, D. Transport Layer Security (TLS) Extensions: Extension Definitions. RFC 6066, January 2011.

[8] Fotiou, N., Marias, G. F., and Polyzos, G. C. Access control enforcement delegation for Information-Centric Networking architectures. ACM SIGCOMM Computer Communication Review 42, 4 (October 2012), 497-502.

[9] Fotiou, N., And Polyzos, G. C. Enabling NAME-Based Security and Trust. In Trust Management IX, C. D. Jensen, S. Marsh, T. Dimitrakos, and Y. Murayama, Eds., vol. 454 of IFIP Advances in Information and Communication Technology. Springer International Publishing, 2015, pp. 47-59.

[10] Fotiou, N., and Polyzos, G. C. Decentralized name-based security for content distribution using blockchains. In Proc. Workshop on Multimedia Streaming in Information- /Content-Centric Networks (MuSIC), in conjuction with IEEE INFOCOM (April 2016).

[11] Green, M., And Ateniese, G. Identity-based proxy re-encryption. In Applied Cryptography and Network Security, J. Katz and M. Yung, Eds., vol. 4521 of Lecture Notes in Computer Science. Springer, 2007, pp. 288-306.

[12] HEss, F. Efficient identity based signature schemes based on pairings. In Selected Areas in Cryptography, K. Nyberg and H. Heys, Eds., vol. 2595 of Lecture Notes in Computer Science. Springer, 2003, pp. 310-324.

[13] Hoffman, P., And Schlyter, J. The DNS-Based Authentication of Named Entities (DANE) Transport Layer Security (TLS) Protocol: TLSA. RFC 6698, August 2012.

[14] Ion, M., Zhang, J., And Schooler, E. M. Toward content-centric privacy in icn:
Attribute-based encryption and routing. In Proc. ACM SIGCOMM Workshop on Information-centric Networking (August 2013), pp. 39-40.

[15] Jacobson, V., Smetters, D. K., Thornton, J. D., Plass, M. F., Briggs, N. H., AND Braynard, R. L. Networking named content. In Proc. ACM International Conference on Emerging Networking Experiments and Technologies (December 2009), pp. 1-12.

[16] Jahid, S., Mittal, P., and Borisov, N. Easier: Encryption-based access control in social networks with efficient revocation. In Proc. ACM Symposium on Information, Computer and Communications Security (March 2011), pp. 411-415.

[17] Kallahalla, M., Riedel, E., Swaminathan, R., WAng, Q., AND Fu, K. Plutus: Scalable secure file sharing on untrusted storage. In Proc. 2nd USENIX Conference on File and Storage Technologies (2003), pp. 29-42.

[18] Li, B., Verleker, A. P., Huang, D., Wang, Z., AND ZHU, Y. Attribute-based access control for icn naming scheme. In Proc. IEEE Conference on Communications and Network Security (October 2014), pp. 391-399.

[19] NaOR, M., AND Pinkas, B. Efficient trace and revoke schemes. In Financial Cryptography, Y. Frankel, Ed., vol. 1962 of Lecture Notes in Computer Science. Springer, 2001, pp. 1-20.

[20] Nilizadeh, S., Jahid, S., Mittal, P., Borisov, N., AND Kapadia, A. Cachet: A decentralized architecture for privacy preserving social networking with caching. In Proc. ACM International Conference on Emerging Networking Experiments and Technologies (December 2012), pp. 337-348.

[21] Tang, Q., Hartel, P., and Jonker, W. Information security and cryptology. M. Yung, P. Liu, and D. Lin, Eds., vol. 5487 of Lecture Notes In Computer Science, Springer-Verlag, pp. 332-347.

[22] Wood, C. A., and Uzun, E. Flexible end-to-end content security in CCN. In Proc. IEEE Consumer Communications and Networking Conference (January 2014), pp. 858-865.

[23] Zhang, X., Chang, K., Xiong, H., Wen, Y., ShI, G., AND Wang, G. Towards name-based trust and security for content-centric network. In Proc. IEEE International Conference on Network Protocols (October 2011), pp. 1-6.

[24] Zheng, Q., Wang, G., Ravindran, R., and AzGin, A. Achieving secure and scalable data access control in information-centric networking. In Proc. IEEE International Conference on Communications (June 2015), pp. 5367-5373. 